\title{
Research on Reliability Prediction of Power Management Module
}

\author{
Shuai $\operatorname{Han}^{1, a}$ and Feng Ding ${ }^{1, b^{*}}$ \\ ${ }^{1}$ Department of Mechanical and Electronic Engineering, Xi'an Technological University, Xi'an, China \\ a13462208735@163.com, bdfeng@xatu.edu.cn \\ *The corresponding author
}

Keywords: Reliability prediction; The component count method; The stress analysis method; The power management module

\begin{abstract}
As an important part of the reliability design, the reliability prediction of electronic products reflects the reliability level of electronic components and the whole system. By analysis the structure and function model of a power management module, this paper separately applies the component count method and the stress analysis method to predict its reliability according to the military standard GJB/Z99C 2006. The analysis results indicate that the component count method is suitable for the preliminary design phase of product due to its simplicity and less time consuming. Furthermore, the stress analysis method is better to be applied in the detailed design phase of product due to its complexity and more time consuming.
\end{abstract}

\section{Introduction}

Power management module (PMM) plays an important role to provide electric power and power management for other modules in the chassis. Therefore it has great significance to carry out the reliability prediction of PMM in a comprehensive way. And as the basic composition of PMM, the quality of components directly affects the overall reliability of the PMM. Many production practices and quality tests show that the quality and reliability of electronic products components have always been an important factor to restrict the quality and service life of products [1]. Therefore, the reliability prediction of PMM components is one of the most important means to evaluate the quality of module.

Since 1950s, the history of reliability prediction of electronic products has been more than 60 years and many new theories and methods are proposed. Luo Mingzhu et al classified the present reliability prediction methods, and drew a conclusion that different reliability prediction methods should be utilized according to the characteristics, life cycle stages and reliability related information of electronic products [2]. Li Xiaomin shows three kinds of reliability prediction methods of the electronic equipment: the component count method, the stress analysis method and the software analysis method[3]. Chen Xiaotong introduced a new method of reliability prediction called PRISM systematically[4]. Guo Lijuan, Li Jinling, Bai Qingyuan et al adopted the stress analysis method to launch the reliability prediction of the electronic meter and the smart meter [5-8]. So far, there are few literatures and standards on the reliability prediction of PMM.

In this paper, the component count method and the stress analysis method are respectively applied to predict reliability of the PMM based on the reliability data and model of its internal components. And the prediction results are helpful to assess the reliability indicators of PMM meet the requirements of design, find the weak link and provide useful data for reconstructing.

\section{Construction of Reliability Model of Power Management Module}

Function, Structure and Characteristics of PMM. PMM is mainly composed of hardware circuit board and LRM module in the form of encapsulation with hot plug, self-examination, control power 
sequence, power management and other functions. And it is used to provide electric power and power management for other modules in the chassis, which is a complex system. The circuit section is divided into three parts: the hot swap unit, the conversion unit and the power management unit. The structure information of the module is shown in Fig.1.

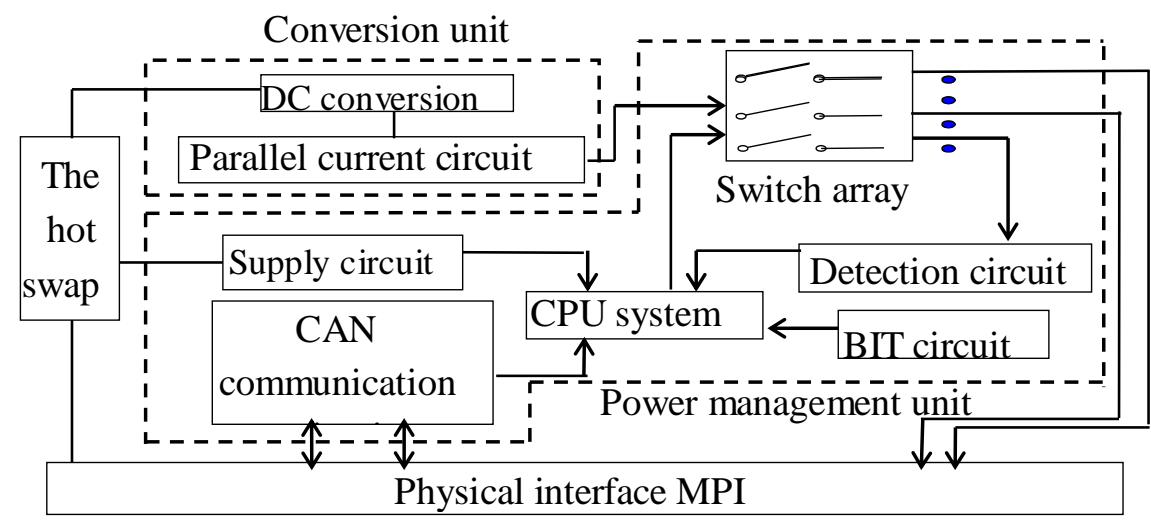

Figure 1. Structure model of power management module

According to the function, the structure and the requirements of its own work of PMM, its characteristics are as follow:

1) Each unit of PMM is approximated by a series relationship. Three units of PMM play an important role in its work, and any one of the unit failure will make the whole PMM paralysis. And it leads to the results that the basic function of PMM cannot be completed. Thus, they are approximated by a series of relationships in the reliability of PMM.

2) The components within each unit of PMM are also approximated by a series relationship (except the power management unit). The power management unit is composed of six parts. And the structure is power supply circuit, CAN communication circuit and BIT circuit form a parallel relationship firstly, then they are in series with the CPU system, PSA and detection circuit. However, the components within each module are also similar to the series relationship.

3) The reliability model of components follows exponential way. The technology of design and manufacture of PMM has been relatively mature, and its components have been screened carefully and have been experienced aging tests. With the exponential distribution and stable working environment, the failure rate of components is constant. So, the failure rate of the module is stable.

Reliability Block Diagram of PMM. By analyzing the function, structure and characteristic of PMM, it is divided into three predicted units: the hot swap unit, the conversion unit and the power management unit. They formed a series model, and the failure rate of PMM is equal to the sum of its three units. The reliability block diagram is shown in Fig. 2.

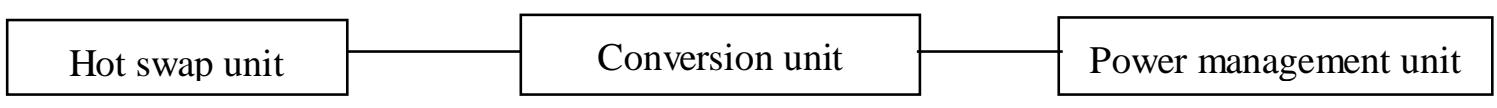

Figure 2. Reliability block diagram of power management module

According to the different research objects, the reliability prediction method of electronic products can be divided into the method based on components, units and equipment system [9]. In order to service PMM, it is necessary to grasp the components to research. Therefore, this paper adopts the component count method and the stress analysis method based on components of PMM to carry on the reliability prediction.

\section{The Reliability Prediction of Power Management Module}

The Foundation of the Theoretical and Data for Prediction. The data investigated in this paper are from the GJB/Z 299C <Handbook of Reliability Prediction of Electronic Equipment > [10]. The 
domestic components follow the data provided in the text and import components follow the data provided in appendix A.

A complete component list of the entire electronic equipment or the product is needed before predicting. PMM consists of 74 types of 269 components and its partial component list is shown in Table 1.

Table 1 The partial component list of power management module

\begin{tabular}{|c|c|c|c|c|c|c|c|}
\hline $\mathrm{S} / \mathrm{N}$ & TYPE & MODEL & Qua & $\mathrm{S} / \mathrm{N}$ & TYPE & MODEL & Qua \\
\hline 1 & MLCC 0805 & 0.1 uf & 41 & 6 & Silicon FEC & CS450S SMD-1 & 1 \\
\hline 2 & Chip ELCO & $10 u / 50 v$ & 4 & 7 & CR & $4.7 \mathrm{~K}$ & 11 \\
\hline 3 & General Diode & LED4 & 1 & 8 & $\mathrm{CR}$ & $35 \mathrm{~K} \quad 1 \%$ & 2 \\
\hline 4 & VR Diode & SY052S 28V-600W & 1 & 9 & MFR & 0.005 & 1 \\
\hline 5 & CR Diode & IN5824 & 2 & 10 & General Diode & LED4 & 1 \\
\hline
\end{tabular}

The Reliability Prediction of Power Management Module with the Component Count Method. The component count method is used to sum the failure rate of the series model based on the types of components. Required information includes the type and quantity of all components, the quality grade of components used and the working environment of the equipment. The calculation formula for the failure rate is:

$$
\lambda_{G S}=\sum_{i=1}^{n} N_{i} \lambda_{G i} \pi_{Q i}
$$

Where $\lambda_{G S}$ is a total failure rate of equipment, $\lambda_{G i}$ represents the general failure rate for $i$ type of components, $\pi_{Q i}$ represents quality factor for $i$ type of components, $N_{i}$ denotes the amount of $i$ type of components and $n$ is the amount of types of components used for the device.

Taking patch capacitor 0805 of model 0.1 uf as an example:

Firstly, the general failure rate $\lambda_{G}$ is determined and the specific type of the component is identified. By seeking information, it can be found that the patch capacitor 0805 of model 0.1 uf belongs to the class I ceramic capacitor. The general environment temperature is selected as $\mathrm{G}_{\mathrm{F} 1}$, according to table $6-22$ by $299 \mathrm{C} \mathrm{GJB} / \mathrm{Z}$ known that $\lambda_{G}=0.025 \times 10^{-6} / \mathrm{h}$.

Secondly, the general quality factor $\pi_{\mathrm{Q}}$ is determined. $\mathrm{A}_{2}$ has been identified as the quality level, according to table 6-4 by $299 \mathrm{C} \mathrm{GJB} / \mathrm{Z}$ known that $\pi_{\mathrm{Q}}=0.3$.

According to the Eq. 1, the failure rate of PMM is $\lambda_{G S}=15.754 \times 10^{-6} / \mathrm{h}$.

In view of the PMM following an exponential distribution, the mean time to failure is $\mathrm{MTTF}=10^{6} / 15.754 \mathrm{~h}=63476 \mathrm{~h} \approx 2644 \mathrm{~d}$.

The Reliability Prediction of Power Management Module with the Stress Analysis Method. In the process of using the stress analysis method to predict the reliability of PMM, the failure rate of components is under the condition of actual applications. In another word, it is the failure rate of components under the influence of various working stress, including working environment temperature, environmental stress, load electrical stress and so on. Required information includes the number of components per type, the failure rate of each component in the reference condition, the stress factor and failure rate conversion model for each component and the structure information of power management module.

The stress analysis method requires PMM is independent in the circuit function, and its reliability model is generally in series structure. As shown in Figure 2, the three prediction units of $\mathrm{PMM}$ are connected in series and the failure rate of PMM is equal to the sum of the failure rates of three units. While calculating the failure rate of the power management unit, the parallel part of the power supply circuit, CAN communication circuit and BIT circuit should be equivalent to the series structure. Then the failure rate of other modules is added to obtain the failure rate of the whole unit. 
The prediction of PMM has 4 steps:

1) Calculating the failure rate of each component of PMM. Analyzing the stress formula of all kinds of components and understanding the effects of the various stress on the reliability of components deeply based on the implementation steps by GJB/Z 299C firstly. Then, compiling the component list of PMM. At last, calculating the failure rate of various components according to the failure rate model of components.

2) Working out the prediction table of the failure rate of components, and putting the failure rate of the 74 types of components calculated from step 1) to fill in the prediction table.

3) Predicting the failure rate of each unit of PMM. Working out the prediction table of units failure rate according to the failure rate of each component determined in step 2). Each prediction unit can be regarded as a series model, and the failure of each component will cause the failure of the prediction unit. So, the failure rate of the unit is equal to the sum of the failure rate of all the components in the unit. The prediction formula is:

$$
\lambda_{p i}=\sum N \lambda_{p}
$$

Where $\lambda_{p}$ is the failure rate of unit $i, \Sigma N \lambda_{p}$ denotes the amount of the failure rate of all the components in the unit $i$.

4) Predicting the failure rate and the mean time to failure of PMM.

The failure rate of three prediction units is shown in Table 2.

Table 2 The failure rate of three prediction unit

\begin{tabular}{cc}
\hline The expected unit & The failure rate $\left[10^{-6} / \mathrm{h}\right]$ \\
\hline The hot swap unit & 0.872 \\
The conversion unit & 3.586 \\
The power management unit & 11.550 \\
\hline
\end{tabular}

So the failure rate of PMM with the stress analysis method is $\lambda_{\mathrm{ps}}=(0.872+3.586+11.550) \times 10^{-6} / \mathrm{h}$ $=16.008 \times 10^{-6} / \mathrm{h}$. And the mean time to failure is MTTF $=10^{6} / 16.008 \mathrm{~h}=62469 \mathrm{~h} \approx 2602 \mathrm{~d}$.

The Results of the Comparison and Analysis of the Two Methods. From the computing process, it can be seen that the component count method is fast and simple. It can be used to accuracy preliminarily judge whether the plan justify the reliability index. However the accuracy of the prediction is not ideal. The stress analysis method is slow and complex. By analyzing the computing result, it can be seen that the mean time to failure of PMM is 2644 days by using the component count method. And the mean time to failure of PMM is 2602 days by using the stress analysis method. Through the stress analysis method, it can accurately reflect the realities of components. For designers, it is helpful to find the reliability weak links of products for improving the design. Meanwhile, designers can get the reliability weak links of products in order to improve the design. From Table 2, it can be observed that the failure rate of the power management unit is relatively large. Thus, in the process of selecting and testing of PMM, the components of the power management unit should be paying more attention.

\section{Conclusion}

The reliability prediction is a key point for the transformation from qualitative analysis to quantitative analysis of electronic products. Through the reliability prediction, we can get more economical and effective data. The weak links can be found and improvement measures are able to be put forward for enhancing the quality and the service life of the product. In this paper, the reliability theory has been researched and we apply it to PMM with two methods. The analysis results demonstrate that the component count method is suitable for the preliminary design phase of 
the product plan, and the stress analysis method is more accurate than the results of the counting method. Thus, it is fit for the detailed design stage of the product plan.

\section{Acknowledgements}

This research is financially supported by the National Science Foundation of China (grant no. 51275374) and the Fund Project of Science \& Technology on Reliability \& Environmental Engineering Key Laboratory.

\section{References}

[1] N. Ma: Practical basic knowledge of electronic products reliability, The Technology and Culture Forum, (2011) No.29, p.144, (In Chinese)

[2] N.Z. Luo, R. Kang and F.W. Liu: A review of reliability prediction methods for electronic products, Electronic Science and Technology, (2014) No.02, p.246, (In Chinese)

[3] X.M. Li, M.F. Wei, J.Y. Qiu and X.L. Zhu: Research on the reliability prediction of electronic equipment, Network New Media and Technology, (2013) No.04, p.48, (In Chinese)

[4] X.T. Chen: Reliability prediction method -- brief introduction of PRISM, Quality and Reliability, (2014) No.05, p.47, (In Chinese)

[5] X.T. Chen: Reliability prediction method -- brief introduction of PRISM, Quality and Reliability, (2014) No.05, p.47, (In Chinese)

[6] J.L. Li and J.L. Guan: Based on the component stress method electric energy meter reliability report, Electronic Components and Applications, (2012) No.6, p.4, (In Chinese)

[7] B.Y. Qing, Y.B. Zhou and G. LI: Reliability forecast of electronic energy meter based on component stress method, Guangxi Electric Power, (2011) No.5, p.19, (In Chinese)

[8] L.J. GUO, Y.Q. LIU, H.J.XU and H. Zeng: Intelligent electric energy meter reliability prediction research based on component stress method, Computer Simulation, (2014) No.1, p.149, (In Chinese)

[9] J.W. Hao: Research on the Methods of Reliability Prediction for the Smart Meter (MS., Henan University of Technology, China2014), (In Chinese)

[10]PLA General Armament Department: <Handbook of Reliability Prediction of Electronic Equipment (Beijing: Department of General Equipment Department of the Ministry of publication and distribution, China 2007), (In Chinese) 